ENFOQUE DEL DERECHO A LA SALUD MENTAL SEGÚN EL TRIBUNAL CONSTITUCIONAL EN CONCORDANCIA CON LA NUEVA LEY DE SALUD MENTAL: UN ANÁLISIS A PARTIR DE UNA SENTENCIA DEL TRIBUNAL CONSTITUCIONAL PERUANO EN CONCORDANCIA CON LA NUEVA LEY DE SALUD MENTAL

FOCUS OF THE RIGHT TO MENTAL HEALTH ACCORDING TO THE CONSTITUTIONAL COURT IN COMPLIANCE WITH THE NEW MENTAL HEALTH LAW: AN ANALYSIS FROM A SENTENCE OF THE PERUVIAN CONSTITUTIONAL COURT IN CONCORDANCE WITH THE NEW MENTAL HEALTH LAW.

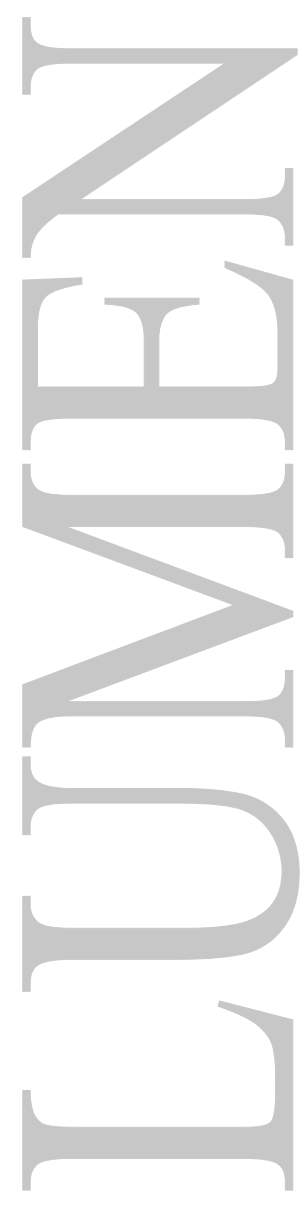




\title{
ENFOQUE DEL DERECHO A LA SALUD MENTAL SEGÚN EL TRIBUNAL CONSTITUCIONAL EN CONCORDANCIA CON LA NUEVA LEY DE SALUD MENTAL: UN ANÁLISIS A PARTIR DE UNA SENTENCIA DEL TRIBUNAL CONSTITUCIONAL PERUANO EN CONCORDANCIA CON LA NUEVA LEY DE SALUD MENTAL
}

\author{
FOCUS OF THE RIGHT TO MENTAL HEALTH ACCORDING TO THE \\ CONSTITUTIONAL COURT IN COMPLIANCE WITH THE NEW MENTAL \\ HEALTH LAW: AN ANALYSIS FROM A SENTENCE OF THE PERUVIAN \\ CONSTITUTIONAL COURT IN CONCORDANCE WITH THE NEW MENTAL \\ HEALTH LAW
}

Beatríz Franciskovic Ingunza

\section{RESUMEN}

El presente comentario de jurisprudencia describe una sentencia del Tribunal Constitucional peruano respecto a un caso en el que se alega la violación al Derecho de Salud mental. Se analizará qué se entiende por Derecho a la Salud, la salud mental, la regulación al respecto que existe en el Perú hasta la nueva Ley de Salud mental así como la función o labor del Estado frente a este derecho fundamental.

\section{PALABRAS CLAVE}

Derecho a la salud, derecho a la salud mental, Tribunal Constitucional peruano, Ley de Salud Mental

\begin{abstract}
The present jurisprudential comment describes a judgment of the Peruvian Constitutional Court regarding a case in which the violation of the Law of Mental Health is alleged. It will be analyzed what is meant by the Right to Health, mental health, the regulation in this respect that exists in Peru until the new Law of Mental Health, as well as the function or work of the State in front of this fundamental right
\end{abstract}

\section{KEY WORDS}

Right to health, right to mental health, Peruvian Constitutional Court

\section{INTRODUCCIÓN}

Por medio del presente comentario se ha analizado una sentencia del Tribunal Constitucional peruano. La mencionada sentencia fue expedida dentro de un proceso constitucional de amparo, basándose en la lesión, afectación y vulneración del Derecho a la Salud mental.

Los hechos a tener en cuenta consisten en que la representante de la demandante solicita se deje sin efecto la atención de alta, pues considera que dicha decisión vulnera el derecho de salud de su representada. Afirma que requiere de una mayor atención y protección, pues de lo contrario podrían atentar contra su vida, de sus representantes y familiares.

Para mejor comprender hemos efectuado un resumen del proceso, señalando los hechos jurídicos relevantes de la parte demandante, la parte demandada, la resolución del Juez, la resolución de los Jueces de la Corte Superior hasta la decisión del Tribunal Constitucional, así como una noción general, alcances y contenido del derecho a la salud, del derecho a la salud mental, así como la regulación de derecho a la salud mental en el Perú. 


\section{HECHOS JURÍDICOS RELEVANTES}

\subsection{HECHOS JURÍDICOS DE LA DEMANDA}

Con fecha 21 de diciembre del año 2005 la demandante en su calidad de curadora de su hija interpone demanda de amparo contra ESSalud, solicitando que se deje sin efecto la orden de alta de su hija G. R. S. (de 46 años), expedida por el Hospital 1 Huariaca ESSalud - Pasco, la misma que padece de esquizofrenia paranoide, y que se le otorgue atención médica permanente e indefinida en dicho centro hospitalario, alegando que dicha orden de alta es violatoria del derecho a la salud.

Señala que el 27 de octubre del año 2004 se le notificó la Carta N. ${ }^{\circ}$ 14-JEDR-CRIPC-HIHESSALUD-04 mediante la cual se informa que su hija se encuentra en condición de alta. En dicha carta se establecen una diversidad de requerimientos y cuidados para recuperar su salud mental y continuar con el tratamiento, algo que es imposible que pueda asumir toda vez que es una anciana que vive sola y en un lugar que carece de servicios básicos como energía eléctrica y agua potable.

Situación que ha sido conocida por la asistenta social del Instituto de Salud Mental en la que se encuentra internada su hija G. R. S.

Su hija es una incapaz absoluta ${ }^{1}$, declarada interdicta civil por padecer de esquizofrenia paranoide de tipo crónico. Es una persona que depende tanto física como mentalmente, por lo que aduce que cuenta con legitimidad procesal ya que la ley le ha conferido la representación de su hija G. R. S.

De otro lado sostiene que debe tomarse en consideración que la enfermedad que padece su hija puede implicar que reaccione con violencia y que pueda atentar contra la vida o la integridad de otras personas, entre ellas, sus propios familiares

\subsection{HECHOS JURÍDICOS RELEVANTES DE LA CONTESTACIÓN DE DEMANDA}

Dentro del plazo de ley, "el Seguro Social de Salud EsSalud cumple con contestar la demanda señalando que la condición de alta de G. R. S. ha sido establecida bajo un criterio médico que ha tomado en consideración el comportamiento y evolución de la paciente.

Asimismo, manifiesta que el padre de G. R. S., ya fallecido, se comprometió a retirar a G. R. S. cuando se disponga su alta, compromiso que es conocido por la recurrente. Por otra parte, argumenta que no se ha adjuntado documento alguno que pruebe que la demandada haya violado el derecho a la salud de la paciente; que las afirmaciones de la demandante, en el sentido de que carece de familiares en el país que puedan hacerse cargo de G. R. S., no deben ser consideradas como una causal que justifique su hospitalización indefinida, pues ha cumplido satisfactoriamente con el tratamiento, encontrándose apta para continuarlo fuera del centro hospitalario

"Sostiene que la enfermedad psiquiátrica de G. R. S. no es pasible de una recuperación total, pero sí cabe la posibilidad de que se reinserte tanto en la sociedad como en la vida familiar, luego de un tratamiento al interior de una institución de salud mental.

\footnotetext{
${ }^{1}$ Al respecto, de conformidad con el Decreto Legislativo 1384 del 04 de setiembre de 2018 solo son considerados como incapaces absolutos los menores de 16 años, salvo para aquellos actos determinados por la ley. Artículo $42^{\circ}$. Capacidad de ejercicio plena. Toda persona mayor de dieciocho años tiene plena capacidad de ejercicio. Esto incluye a todas las personas con discapacidad, en igualdad de condiciones con las demás y en todos los aspectos de la vida, independientemente de si usan o requieren de ajustes razonables o apoyos para la manifestación de su voluntad. Excepcionalmente, tienen plena capacidad de ejercicio los mayores de catorce años y menores de dieciocho años que contraigan matrimonio, o quienes ejerciten la paternidad.
} 


\subsection{RESOLUCIÓN JUDICIAL DEL DÉCIMO JUZGADO ESPECIALIZADO EN LO CIVIL DE LIMA}

Con fecha 2 de mayo del año 2006, el Décimo Juzgado Especializado en lo Civil de la Corte Superior de Justicia de Lima declara improcedente la demanda, por considerar que no se ha producido una vulneración o amenaza contra el derecho a la salud de la recurrente; por lo que, en todo caso, la determinación de esta afectación o amenaza del derecho fundamental requeriría de la actuación de medios probatorios, lo que no resulta viable en un proceso constitucional de amparo, en virtud de lo dispuesto en el artículo 9. ${ }^{\circ}$ del Código Procesal Constitucional. No conforme con lo expuesto, la parte demandante interpone recurso de apelación".

\subsection{RESOLUCIÓN DE LA QUINTA SALA CIVIL DE LA CORTE SUPERIOR DE LIMA}

El 26 de marzo de 2017, la Quinta Sala Civil de la Corte Superior de Lima confirma la resolución apelada señalando que no existen elementos de juicio suficientes para considerar que se ha incumplido con el deber del Estado de otorgar una prestación integral de salud a G. R. S. toda vez que la demandada no ha suspendido el tratamiento a la paciente, sino que considera que ésta se encuentra en condiciones de seguirlo en condición de alta. Asimismo, expresa que si lo que se pretende es cuestionar el informe médico debido a que no se corresponde con el estado real de la paciente, ello requerirá de una pericia médica, lo no puede ser determinado en un proceso de amparo ya que carece de etapa probatoria, conforme a lo dispuesto por el artículo $9 .{ }^{\circ}$ del Código Procesal Constitucional.

\subsection{RECURSO DE AGRAVIO CONSTITUCIONAL}

La demandante no conforme con la resolución de vista, el día 27 de abril del año 2007, interpone recurso de agravio constitucional.

Afirma que en el propio informe médico de alta se sostiene que la paciente no va a presentar una mejoría mayor con el tiempo, entonces lo que realmente están pretendiendo la parte demandada es desprenderse de su hija, lo que constituye una afectación del derecho a la salud.

Discrepa de la necesidad de la existencia de una etapa probatoria, en la medida que la vulneración del derecho a la salud es evidente, ya que el propio informe médico reconoce que no se producirá una mayor mejoría con el tiempo.

Por otro lado, afirma que resulta evidente que la entrega de la paciente a R. J. S. A. Vda. de R. pondría en serio riesgo su vida e integridad física y psíquica.

\subsection{RESOLUCIÓN DEL TRIBUNAL CONSTITUCIONAL:}

Según el Tribunal Constitucional se encuentra acreditada la imposibilidad de la madre de la paciente G.R.S. de poder hacerse cargo de su hija debido a su avanzada edad, y advierte también la necesidad de que una persona lleve un control del tratamiento farmacológico que como lo ha expresado el amicus curiae, ha de ser por tiempo indefinido.

Es necesario disponer, por tanto, que las autoridades del centro hospitalario donde se encuentra la paciente brinden de por vida las atenciones médicas necesarias para que no haya retrocesos en el nivel de desarrollo de la enfermedad de la cual adolece G. R. S. por lo que en consecuencia, se declara fundada la demanda de amparo, qué se deje sin efecto el informe de alta de fecha 7 de noviembre de 2005, expedido por el Hospital 1 Huariaca-EsSalud - Pasco, y Ordenar que el Seguro Social de Salud-EsSalud, otorgue a G.R.S., atención médica y hospitalización permanente e indefinida, y la provisión constante de medicamentos necesarios para el tratamiento de su enfermedad mental, así como la realización de exámenes periódicos. 
Del contenido de cada una de las resoluciones previas a la sentencia del Tribunal Constitucional se puede señalar que no se está de acuerdo con lo resuelto ni por el juez de primera instancia ni con lo de la corte superior. Los jueces citados, al resolver, no han tenido en cuenta que el derecho a la salud, específicamente el derecho de la salud mental, constituye un derecho fundamental que no tiene un pronóstico final determinado. El Estado, no puede dejar de otorgar prestación integral de salud. Pues, el tratamiento de una enfermedad mental como la esquizofrenia requiere de un proceso continuo, permanente e indefinido de medicamentos, es decir, el procedimiento de dicha enfermedad no puede concluir con un informe de alta, dando por concluida dicha atención sobre todo cuando la misma señala una variedad de requerimientos y cuidados para que la paciente recupere su salud mental.

Por el contrario, se rescata y aplaude la decisión del Tribunal Constitucional que al resolver toma en consideración la edad, economía y circunstancias de la representante así como la salud, tratamiento y enfermedad mental de la representada, ordenando que se deje sin efecto el informe de alta y que el Seguro Social de Salud-EsSalud, se le otorgue atención médica y hospitalización permanente e indefinida así como la provisión constante de medicamentos necesarios para el tratamiento de su enfermedad mental así como la realización de exámenes periódicos.

\section{EL DERECHO A LA SALUD.}

\subsection{QUÉ SE ENTIENDE POR EL TÉRMINO O EXPRESIÓN SALUD}

Expuestos los hechos jurídicos relevantes que dieron origen a la presente sentencia resulta importante determinar qué se entiende por el término o expresión salud.

"La salud puede ser entendida como el funcionamiento armónico del organismo tanto del aspecto físico como psicológico del ser humano. Es evidente que como tal constituye una condición indispensable para el desarrollo y medio fundamental para alcanzar el bienestar individual y colectivo". (Azanca con Ministerio de Salud , 2003 )

La expresión salud se encuentra relacionada con el buen funcionamiento íntegro del cuerpo del ser humano. Es decir, con el funcionamiento correcto e idóneo del ser humano, entendido como una unidad inseparable de mente, cuerpo (físico y biológico) y espíritu. Con esto se alcanza un bienestar de toda la sociedad en su conjunto.

En el año 1948 la Organización Mundial de la Salud - OMS promulgó una definición clásica de salud, señalando que es el completo estado de bienestar físico, mental y social y no solamente la ausencia de afecciones o enfermedades. "A partir de esta definición han surgido nuevas concepciones de este proceso, asumiendo una multideterminación en su origen, factores económicos, políticos, sociales, culturales, biológicos, de servicios de salud, del entorno. Hoy impera una nueva visión, es un entendimiento de la salud bajo la perspectiva de la vida y es este un redimensionamiento del concepto salud-enfermedad" (Azanca con Ministerio de Salud, 2003 )

"Aunque existen decenas de definiciones sobre el concepto de salud, la mayoría de ellas coincide en que este proceso es finalmente una manifestación de la capacidad de adaptación del hombre a su ambiente" (Vélez Arango , 2007, págs. 63-78)

Salud es un concepto amplio, que "incluye diversas interpretaciones subjetivas de lo que significa "sentirse bien" y que por cierto es mucho más amplio que simplemente "no sufrir de enfermedad alguna". (Rondón, 2006, pág. 237)

La expresión salud no puede ser entendida únicamente como aquel estado de no estar enfermo o no padecer enfermedad alguna, sino que constituye un estado mayor, de bienestar general y bienestar en todos los aspectos de un ser humano, bienestar en lo físico, en lo biológico, en lo 
mental y hasta en lo espiritual que se manifiesta con la quietud y tranquilidad de alma y de buen corazón.

Guillermo Escobar Roca sostiene que "la salud es el bien jurídico constitucional que da vida al derecho a la protección de la salud (como se le denomina en España), o al derecho a la salud. En ese sentido, afirma que la salud es un bien jurídico constitucional al que puede considerársele el objeto o finalidad del derecho, pero no estrictamente su contenido. El contenido del derecho a la salud no viene conformado por la salud en sí sino por las acciones encaminadas a su consecución" (Ticona Cano, 2014 , pág. 31)

\subsection{NOCIÓN Y CONTENIDO DEL DERECHO A LA SALUD}

El derecho a la salud constituye un derecho fundamental. Como todo derecho fundamental es inherente a todo ser humano, imprescriptible, inalienable, irrenunciable y absoluto, salvo las limitaciones establecidas por el Estado.

Sin duda, como todo derecho subjetivo y fundamental se encuentra relacionado con el valor de dignidad, libertad e igualdad.

"El contenido mínimo esencial para el derecho a la salud se refiere al acceso universal a bienes y servicios de salud, a medicamentos esenciales, a asistencia médica y sanitaria preventiva y curativa, a un plan nacional de salud pública, a la provisión de seguro y de servicios de la salud para quienes carecen de recursos; además a la educación e información en salud y a la cooperación internacional". (Echeverri L, 2016, pág. 88)

“(...) el derecho a la salud comprende la facultad que tiene todo ser humano de mantener el estado de normalidad orgánica funcional, tanto física como mental, y de restablecerse cuando se presente una perturbación en la estabilidad orgánica y funcional de su ser, lo que implica, por tanto, una acción de conservación y otra de restablecimiento; acciones cuyo cumplimiento corresponde al Estado, el cual debe garantizar una progresiva y cada vez más consolidada calidad de vida, invirtiendo en la modernización y fortalecimiento de todas las instituciones encargadas de la prestación del servicio de salud, debiendo, para tal efecto, adoptar políticas, planes y programas en ese sentido". (Azanca con Ministerio de Salud , 2003 )

Según el Tribunal Constitucional la salud no puede ser entendida desde un ámbito negativo de ausencia de enfermedad, sino que exige también una contraparte, una faceta positiva, en el sentido de que todos tienen (...) el derecho de que se le asignen medidas sanitarias y sociales relativas a la alimentación, vestido, vivienda y asistencia médica, correspondiente al nivel que lo permiten los recursos públicos y la solidaridad de la comunidad.

En este orden de ideas el Tribunal Constitucional considera que la salud puede ser enfocada desde tres perspectivas: a) individual, b) familiar y c) en un contexto comunitario o colectivo. Este último aspecto -el comunitario o colectivo- trae como consecuencia directa que su exigibilidad se vea condicionada a aspectos presupuestales

Por derecho a la salud se entiende "el acceso a los servicios de promoción de la salud, de prevención y curación de enfermedades y de rehabilitación en los casos de deterioro de la salud. Esta categoría abarca también el acceso a una alimentación adecuada, a condiciones de trabajo sanas y seguras y a servicios de apoyo para el cuidado de la salud". (Alvarez Castaño, 2005, pág. 134)

El derecho a la salud significa que todo el mundo, que todos los sujetos de derecho deben tener acceso a los servicios de salud cuándo y dónde los necesite, sin tener que hacer frente a dificultades económicas. Nadie debería enfermarse o morir por ser pobre o porque no pueda acceder a los servicios de salud que necesita. Resulta evidente que la salud también se ve condicionada por otros derechos 
humanos fundamentales, como el derecho al agua potable, el derecho a una vida en condiciones dignas, el acceso a la educación y al trabajo. También hace referencia al derecho a la integridad, vida y salud sobre su cuerpo, entendido éste como una unidad inseparable de mente, cuerpo y espíritu.

"La salud pública, por su esencia, va más allá de la persona. [...] En ese sentido, la naturaleza se funda con el ser humano para su bienestar; particularmente, si consideramos que la salud mental, física y social están interconectadas y son vitales para los individuos. Al respecto, la salud mental debería ser una preocupación para todos nosotros, en lugar de solo para aquellos que sufren un trastorno mental". (Estay Sepúlveda, Juan Guillermo; Crespo, Julio; Lagomarsino Montoya, Mario; Peña Testa, Claudia., 2018, pág. 43)

\subsection{NOCIÓN DE SALUD MENTAL: ¿QUÉ SE ENTIENDE POR SALUD MENTAL?}

Por salud mental hay que entender y comprender aquel estado de quietud, sosiego y tranquilidad en lo sentimental, psicológico, afectivo y social.

La salud mental incluye el buen estado emocional, social y psicológico. Influye en la forma que uno piensa, cómo siente y cómo se comporta frente a los demás.

La salud mental, según la definición propuesta por la OMS es el "estado de bienestar que permite a cada individuo realizar su potencial, enfrentarse a las dificultades usuales de la vida, trabajar productiva y fructíferamente y contribuir con su comunidad. Si analizamos esta definición, notaremos que la falta de salud mental impide que las personas lleguen a ser lo que desean ser, no les permite superar los obstáculos cotidianos y avanzar con su proyecto de vida y da lugar a falta de productividad y de compromiso cívico con la nación. El resultado: frustración, pobreza y violencia, junto con la incapacidad de desarrollar en democracia y de considerar el bien común". (Rondón, 2006, pág. 237)

La salud mental se puede entender como "un proceso dinámico que, generalmente, ocurre en un continuo salud-enfermedad mental, aunque en la mayoría de los casos no se llega necesariamente a la enfermedad y estas personas pueden mantener su salud mental con variaciones dependientes de diversas circunstancias. Las personas que no padecen de trastorno mental pueden presentar otro tipo de problemas de salud mental que pueden afectar su bienestar emocional, así como problemas psicosociales que afectan su funcionamiento y por ende su vida cotidiana. De ahí que la salud mental condicione las actividades que realizan las personas y habilidades psicosociales que tienen para enfrentarse a los problemas de la vida cotidiana. Cuando las personas no han adquirido las habilidades psicosociales necesarias, tendrán dificultades para desempeñarse satisfactoriamente en las diversas áreas de sus funciones por lo que pueden presentar problemas en una ocupación o trabajo, en el manejo de sus ingresos económicos, en sus relaciones sociales, en sus relaciones de pareja, incluido su componente sexual y en sus relaciones familiares" (Valencia Collazos, 2007, pág. 78)

"Las enfermedades mentales son causa de gran sufrimiento personal para quienes las padecen y para las personas de su entorno. En un país donde la atención psiquiátrica no está disponible en varias regiones y donde los seguros privados no están obligados por ley a cubrir tales atenciones, la enfermedad mental empobrece rápidamente a la familia. Por otro lado, la falta de un sistema de atención y rehabilitación con base en la comunidad, obliga a que pacientes con síntomas graves, que dificultan significativamente la interacción familiar, deban vivir y permanecer todo el día en sus casas, el resultado es el rechazo familiar, la discriminación y exclusión del enfermo y del familiar que lo cuida, con graves consecuencias para la salud mental del cuidador"' (Rondón, 2006, pág. 238)

Cabe destacar que la expresión mental, en la definición de salud de la OMS (así como físico y social) se refiere a "dimensiones de un estado y no a un dominio o disciplina específicos. 
En consecuencia, conforme a este concepto, resulta incongruente hablar de salud física, salud mental o salud social. Si quisiéramos especificar una dimensión en particular, sería más apropiado utilizar el término bienestar y no salud (por ejemplo, bienestar mental o bienestar social). Este uso negligente de la palabra salud parece haberse iniciado cuando se sustituyó la higiene mental (movimiento social o dominio de actividad) por salud mental (originalmente destinado a un estado y después transformado en un dominio o campo de actividad). (Bertolote, 2008, pág. 114) «La salud mental es considerada como una condición que permite el desarrollo físico, intelectual y emocional óptimo de un individuo, en la medida que ello sea compatible con la de otros individuos" (Ticona Cano, 2014 , pág. 31)

La salud mental, "puede ser enunciada como la construcción y desarrollo o deterioro en las distintas prácticas sociales, en el trabajo, la vida familiar, comunitaria, en la política y en lo social. Salud mental en una comunidad es un proceso resultante de sus condiciones de vida, de su historia y de sus proyectos. En síntesis, la salud mental se asocia a la vivencia de satisfacción tanto material como simbólica, y se evidencia en la construcción de vínculos sanos, creativos y solidarios; en la participación real en las decisiones que afectan la vida cotidiana de los sujetos; en la posibilidad de pertenencia, integración y construcción de sus proyectos. En la práctica cotidiana ello implica un sujeto crítico, reflexivo, pensante, flexible y creativo que sepa construir las estrategias y las herramientas posibles necesarias para enfrentar los procesos y situaciones en forma constructiva y colectiva. (Custo, 2008, pág. 22)

"La salud mental consiste en el aprendizaje de la realidad para transformarla a través del enfrentamiento, manejo y solución integradora de conflictos, tanto internos del individuo como los que se dan entre él y su medio. Cuando dicho aprendizaje se perturba o fracasa en el intento de su resolución, puede comenzarse a hablar de enfermedad. En sentido similar, Pichón Riviere apunta: la salud mental se concibe como la capacidad de mantener con el mundo relaciones dialécticas y transformadoras que permitan resolver las contradicciones internas del individuo y las de éste con el contexto social." (Oramas Viera, Arlene; Santana López, Sandra; Vergara Barrenechea, Adriana, 2006 , pág. 34)

\subsection{EL DERECHO A LA SALUD MENTAL EN EL PERÚ}

El derecho a la salud mental en el Perú se encuentra expresamente establecido en el artículo $7^{\circ}$ de la Constitución Política del Perú , la misma que consagra el derecho de toda persona "(...) a la protección de su salud, la del medio familiar y la de la comunidad, así como al deber de contribuir a su promoción y defensa. La persona incapacitada para velar por sí misma a causa de una deficiencia física o mental tiene derecho al respeto de su dignidad y a un régimen legal de protección, atención, readaptación y seguridad".

Además el artículo $9^{\circ}$ de la Ley Fundamental dispone que “(...) el Estado determina la política nacional de salud. El poder ejecutivo norma y supervisa su aplicación. Es responsable de diseñarla y conducirla en forma plural y descentralizadora para facilitar a todos el acceso equitativo a los servicios de salud".

En el Perú el Ministerio de Salud es el ente encargado de regular y supervisar el derecho de los usuarios de los servicios de salud. Fue creado mediante el Decreto Ley Número 8124 con la denominación de Ministerio de Salud Pública, Trabajo y Previsión Social. Posteriormente en el año 1942 adopta el nombre de Ministerio de Salud Pública y Asistencia Social, hasta que en el año 1968 obtiene la denominación que actualmente mantiene.

La Ley del Ministerio de Salud- Ley 27657 del 29 de enero del año 2002 determina que el Ministerio de Salud es una entidad que forma parte del Poder Ejecutivo. Es el ente que conduce, regula y 
promueve la intervención del Sistema Nacional de Salud, con la finalidad de lograr el desarrollo de la persona humana, a través de la promoción, protección, recuperación y rehabilitación de su salud y del desarrollo de un entorno saludable, con pleno respeto de los derechos fundamentales de la persona.

Por su parte, la Ley General de Salud, Ley Número 26842 del 09 de julio de 1997, establece en su título preliminar artículo $\mathrm{I}$, que la salud es condición indispensable del desarrollo humano y medio fundamental para alcanzar el bienestar individual y colectivo. II. La protección de la salud es de interés público. Por tanto, es responsabilidad del Estado regularla, vigilarla y promoverla. III. Toda persona tiene derecho a la protección de su salud en los términos y condiciones que establece la ley. El derecho a la protección de la salud es irrenunciable. El concebido es sujeto de derecho en el campo de la salud. IV. La salud pública es responsabilidad primaria del Estado. La responsabilidad en materia de salud individual es compartida por el individuo, la sociedad y el Estado".

Del mismo modo, el artículo $\mathrm{V}$ de la citada Ley, modificado por la Ley 29973 - Ley general de la persona con discapacidad del 13 de diciembre de 2012 señala: "Es responsabilidad del Estado vigilar, cautelar y atender los problemas de desnutrición y de salud mental de la población, y los de salud ambiental, así como los problemas de salud de la persona con discapacidad, del niño, del adolescente, de la madre y del adulto mayor en situación de abandono social".

Siguiendo con lo establecido por la Ley General de Salud, se establece que es de interés público la provisión de servicios de salud, cualquiera sea la persona o institución que los provea. Es responsabilidad del Estado promover las condiciones que garanticen una adecuada cobertura de prestaciones de salud a la población, en términos socialmente aceptables de seguridad, oportunidad y calidad. IX La norma de salud es de orden público y regula materia sanitaria, así como la protección del ambiente para la salud y la asistencia médica para la recuperación y rehabilitación de la salud de las personas.

El "artículo 3 precisa: Toda persona tiene derecho a recibir, en cualquier establecimiento de salud, atención médico-quirúrgica de emergencia cuando la necesite y mientras subsista el estado de grave riesgo para su vida o su salud. Artículo 9. Toda persona que adolece de discapacidad física, mental o sensorial tiene derecho al tratamiento y rehabilitación. El Estado da atención preferente a los niños y adolescentes. Las personas con discapacidad severa, afectadas además por una enfermedad, tienen preferencia en la atención de su salud".

El artículo 11 de la Ley General de Salud ha sufrido varias modificaciones (una primera modificación, mediante la Ley Nro. 29737 del 06 de julio de 2011, referida a la salud mental y que reguló los procedimientos de internamiento de las personas con trastornos mentales; segunda modificación, el día 24 de junio de 2012 por la Ley 29889 - Ley que garantiza los derechos de las personas con problemas de salud mental; la misma que ha sido derogada, mediante la única disposición complementaria derogatoria de la reciente Ley de Salud Mental - Ley 30947 del 23 de mayo de 2019)

Además de los procedimientos y derechos establecidos en el artículo 15 de la presente Ley, en la atención de la salud mental se considera lo siguiente: a. La atención de la salud mental se realiza en el marco de un abordaje comunitario, interdisciplinario, integral, participativo, descentralizado e intersectorial. b. La atención de la salud mental se realiza preferentemente de manera ambulatoria, dentro del entorno familiar, comunitario y social. c. El internamiento es un recurso terapéutico de carácter excepcional y solo puede llevarse a cabo cuando aporte mayores beneficios terapéuticos para el paciente que el resto de intervenciones posibles. Se realiza por el tiempo estrictamente necesario y en el establecimiento de salud más cercano al domicilio del usuario. $d$. El tratamiento e internamiento se realizan con el consentimiento informado, libre y voluntario del usuario, salvo 
en situaciones de emergencia. e. La revisión médica periódica de los diagnósticos e informes que recomiendan el internamiento de pacientes. El internamiento tendrá una segunda opinión médica. f. Los usuarios de los servicios de salud mental, incluidas las personas con discapacidad mental, mantienen el pleno ejercicio de sus derechos durante su tratamiento e internamiento. g. Las personas con adicciones gozan de los mismos derechos y garantías que se reconocen a los demás usuarios de los servicios de salud. Su tratamiento e internamiento involuntario no requiere de su consentimiento informado y se realiza a solicitud de la familia cuando su capacidad de juicio esté afectada, lo cual debe ser determinado por una Junta Médica."

Sobre las personas con discapacidad es importante precisar que la Ley General de la Persona con Discapacidad - Ley N $N^{\circ} 29973$ del 13 de diciembre de 2012 en el artículo 2., la define como "aquella que tiene una o más deficiencias físicas, sensoriales, mentales o intelectuales de carácter permanente que, al interactuar con diversas barreras actitudinales y del entorno, no ejerza o pueda verse impedida en el ejercicio de sus derechos y su inclusión plena y efectiva en la sociedad, en igualdad de condiciones que las demás". Precisándose en el artículo 3. Derechos de la persona con discapacidad 3.1 La persona con discapacidad tiene los mismos derechos que el resto de la población, sin perjuicio de las medidas específicas establecidas en las normas nacionales e internacionales para que alcance la igualdad de hecho. El Estado garantiza un entorno propicio, accesible y equitativo para su pleno disfrute sin discriminación. 3.2 Los derechos de la persona con discapacidad son interpretados de conformidad con los principios y derechos contenidos en la Declaración universal de los Derechos Humanos, la Convención sobre los Derechos de las Personas con Discapacidad y con los demás instrumentos internacionales sobre derechos humanos ratificados por el Perú".

Al respecto el 04 de setiembre de 2018 se promulgó el Decreto Legislativo 1384, el mismo que reconoce y regula la capacidad jurídica de las personas con discapacidad en igualdad de condiciones. "Entendemos por discapacidad la dificultad para desempeñar papeles y desarrollar actividades socialmente aceptadas, habituales para las personas de similar edad y condición, es decir la discapacidad es la dificultad o la imposibilidad para llevar a cabo una función o un papel en un contexto social y en un entorno determinado. La discapacidad es la expresión de una limitación funcional, emocional o cognitiva en un contexto determinado. Es la brecha existente entre las capacidades de las personas (condicionadas en parte por su salud) y las demandas del medio (físico, social, laboral). Se vincula más a la función social que a la función orgánica (a la que se asocian patología y deficiencia) estas dificultades obligan a la persona que las sufre a utilizar dispositivos o bien, principalmente, a solicitar ayuda de otra persona para poder realizar esas actividades cotidianas. En eso consiste la dependencia" (Puga, 2005, pág. 328)

El artículo 37, prescribe que: los establecimientos de salud y los servicios médicos de apoyo, cualquiera sea su naturaleza o modalidad de gestión, deben cumplir los requisitos que disponen los reglamentos y normas técnicas que dicta la Autoridad de Salud de nivel nacional. La Autoridad de Salud de nivel nacional o a quien ésta delegue, verificará periódicamente el cumplimiento de lo establecido en la presente disposición. El artículo 44, señala que al egreso del paciente, el responsable del establecimiento de salud está obligado a entregar al paciente o a su representante el informe de alta que contiene el diagnóstico de ingreso, los procedimientos efectuados, el diagnóstico de alta, pronóstico y recomendaciones del padecimiento que ameritó el internamiento. Así mismo, cuando el paciente o su representante lo soliciten, debe proporcionarle copia de la epicrisis y de la historia clínica, en cuyo caso el costo será asumido por el interesado

El 23 de mayo de 2019 ha sido publicada la Ley de Salud Mental - Ley 30947, cuyo objeto es establecer el marco legal para garantizar el acceso a los servicios, la promoción, prevención, tratamiento y rehabilitación en salud mental, como condiciones para el pleno ejercicio del derecho a la salud y el bienestar de la persona, la familia y la comunidad. Se señala que corresponde a los 
Ministerios de Salud, de Educación, de la Mujer y Poblaciones Vulnerables, de Justicia y Derechos humanos, de Trabajo y Promoción del Empleo y de Desarrollo e Inclusión Social así como a los gobiernos regionales, locales y a la sanidad de las Fuerzas Armadas y Fuerzas Policiales, en el ámbito de sus competencias y funciones, a los establecimientos de la salud privados y a otros prestadores, la aplicación y cumplimiento de las disposiciones contenidas en la presente ley. Establece varios principios y enfoques trasversales.

El capítulo II denominado como Derechos en Salud Mental en el artículo 7 señala textualmente que "en el marco de lo establecido por el artículo 7 de la Constitución Política del Perú, toda persona, sin discriminación alguna, tiene derecho a gozar del más alto nivel posible de salud mental. El Estado garantiza la disponibilidad de programas y servicios para la atención de la salud mental en número suficiente, en todo el territorio nacional; así como el acceso a prestaciones de salud mental adecuadas y de calidad, incluyendo intervenciones de promoción, prevención, recuperación y rehabilitación".

En el artículo 10 - atención por los seguros de salud, se prescribe que: "los seguros de salud públicos y privados deben cubrir la atención en salud mental dentro de sus planes, incluyendo el tratamiento ambulatorio o el internamiento u hospitalización, así como el acceso a medicamentos y productos sanitarios adecuados y de calidad, con excepción de los seguros con póliza de naturaleza específica. Las compañías privadas que ofrecen seguros de salud están obligadas a brindar cobertura para el diagnóstico, tratamiento y rehabilitación en salud mental, de acuerdo a las necesidades de las personas con los problemas de salud mental registrados en el Capítulo de Trastornos Mentales y del Comportamiento de la Clasificación Internacional de Enfermedades de la Organización Mundial de la Salud vigente al momento de la atención de salud, con la frecuencia, cantidad y duración necesaria. Las personas en condición de pobreza o pobreza extrema con discapacidad mental que no cuenten con seguros deben ser incluidas en el Seguro Integral de Salud (SIS), de acuerdo a un plan progresivo de atención o prestaciones."

Algo importante de esta Ley y que tiene mucha relación con lo resuelto, con fecha muy anterior por el Tribunal Constitucional, es lo establecido en los artículos del 30 al 33, que en resumen señala 3 supuestos de personas con enfermedad mental que se encuentran hospitalizadas y dependiendo de su situación se prevé una solución. Así se señala:

i) Que las personas que, a pesar de contar con alta médica, por circunstancias ajenas a su voluntad, permanecen innecesariamente hospitalizadas en los establecimientos de salud, estás deben ser derivadas a profesionales de trabajo social o especialidades afines para movilizar la red familiar y comunitaria y promover la reinserción en dichos ámbitos

ii) Que las personas en situación de abandono o desprotección, una vez que sea controlada la crisis que provocó su internamiento u hospitalización, el responsable del establecimiento de salud, en coordinación con el Ministerio de la Mujer y Poblaciones Vulnerables, dispondrá la búsqueda de los familiares de la persona atendida. En caso de que no se ubique a sus familiares, dichas personas son trasladadas a hogares protegidos $\mathrm{y}$, excepcionalmente, a centros de atención residencial del Estado, donde continúan recibiendo el tratamiento ambulatorio a que hubiere lugar en el establecimiento de salud más cercano

En ambos casos, los servicios de salud mental, tratándose de personas en situación de pobreza, víctimas de todo tipo de violencia, minorías étnicas, poblaciones afectadas por desastres naturales, mujeres, niñas, niños y adolescentes y personas de la tercera edad, entre otros; deben tener en consideración dichas necesidades especiales.

iii) Que las personas que tenga un período de internamiento u hospitalización mayor a cuarenta y cinco (45) días y que se encuentre en condición de alta médica, debe continuar su tratamiento en forma ambulatoria y ser incorporada en la red de atención comunitaria de salud, coordinando previamente con los profesionales de trabajo social y afines. 
Sin duda el Tribunal Constitucional ha resuelto en base al contenido y alcances del derecho a la salud mental. Ha respetado los lineamientos y parámetros de las normas al respecto y ha decidido con una visión objetiva (teniendo en cuenta los hechos y datos del presente caso) e imparcial, amparado en las normas citadas. Es deber del Estado en este caso, por medio de esta sentencia, no atentar contra la integridad y salud tanto de la demandante como de su representada. Es deber del Estado otorgar mayor protección y atención a los enfermos de la salud mental, privilegiando el caso en particular y no amparar resoluciones meramente formales, aduciendo que no se ha vulnerado el derecho a la salud, con una visión sesgada sin concederle mayor atención a los hechos del presente caso, es decir, sin tener a la vista la enfermedad que padecía la representada, su avanzada edad así como la edad, salud y economía de su representada, quien realmente se encontraba imposibilitada e impedida de hacerse cargo de su hija, por tratarse de una enfermedad que no tiene cura si no que depende de un tratamiento farmacológico indefinido de por vida.

\section{CONCLUSIONES}

No se está de acuerdo con lo resuelto ni por el Juez de primera instancia ni con lo de la Corte Superior. Los jueces no han tenido en cuenta que el derecho a la salud, específicamente el derecho a la salud mental, constituye un derecho fundamental que no tiene un pronóstico final determinado.

El tratamiento de una enfermedad mental como la esquizofrenia requiere de un proceso continuo, permanente e indefinido de medicamentos, es decir, el procedimiento de dicha enfermedad no puede concluir con un informe de alta, dando por concluido dicha atención sobre todo cuando la misma señala una variedad de requerimientos y cuidados para que la paciente recupere su salud mental.

Por el contrario, se felicita la decisión del Tribunal Constitucional que al resolver toma en consideración la edad, economía y circunstancias de la representante así como la salud, tratamiento y enfermedad mental de la representada, ordenando que se deje sin efecto el informe de alta y que el Seguro Social de Salud-EsSalud, se le otorgue atención médica y hospitalización permanente e indefinida así como la provisión constante de medicamentos necesarios para el tratamiento de su enfermedad mental así como la realización de exámenes periódicos.

El Colegiado del Tribunal Constitucional ha resuelto en base al contenido y alcances del derecho a la salud mental. Ha respetado los lineamientos y parámetros de las normas al respecto y ha decidido con una visión objetiva (teniendo en cuenta los hechos y datos del presente caso) e imparcial, amparado en las normas citadas. Es deber del Estado, en este caso, por medio de esta sentencia, no atentar contra la integridad y salud tanto de la demandante como de su representada. Es deber del Estado otorgar mayor protección y atención a los enfermos de la salud mental, privilegiando el caso en particular y no amparar resoluciones meramente formales, aduciendo que no se ha vulnerado el derecho a la salud, con una visión sesgada sin conceder mayor atención a los hechos del presente caso, es decir, sin tener en consideración la enfermedad que padecía la representada, su avanzada edad así como la edad, salud y economía de su representada, quien realmente se encontraba imposibilitada e impedida de hacerse cargo de su hija, por tratarse de una enfermedad que no tiene cura si no que depende de un tratamiento farmacológico indefinido de por vida.

La expresión salud se encuentra relacionada con el buen funcionamiento íntegro del cuerpo del ser humano. Es decir, con el funcionamiento correcto e idóneo del ser humano, entendido como una unidad inseparable de mente, cuerpo (físico y biológico) y espíritu. Con esto se alcanza un bienestar de toda la sociedad en su conjunto.

El derecho a la salud significa que todo sujeto de derecho debe tener acceso a los servicios de salud cuándo y dónde los necesite, sin tener que hacer frente a dificultades económicas. 
Por salud mental hay que entender y comprender aquel estado de quietud, sosiego y tranquilidad en lo sentimental, psicológico, afectivo y social. La salud mental incluye el buen estado emocional, social y psicológico. Influye en la forma que uno piensa, cómo siente y cómo se comporta frente a los demás.

\section{REFERENCIAS}

- Alcántara Moreno , G. (2008). La definición de salud de la Organización Mundial de la Salud y la interdisciplinariedad. Sapiens. Revista Universitaria de Investigación(1), 93 - 107.

- Alvarez Castaño, L. (2005). El derecho a la Salud en Colombia: una propuesta para su fundamentación moral. Revista Panamericana de Salud Pública, 95, 129-135.

- $\quad$ Azanca con Ministerio de Salud, Tribunal Constitucional Peruano (2003).

- Bertolote, J. (2008). Raíces del concepto de salud mental. (E. Esp, Ed.) World Psychiatry, 113-116.

- Custo, E. (2008). Salud mental y ciudadanía: una perspectiva desde el Trabajo. Buenos Aires: Espacio editorial.

- Echeverri L, M. (2016). Derecho a la Salud, Estado y Globalización. Revista Facultad Nacional de Salud Pública, 24, 81 - 96.

- Estay Sepúlveda, Juan Guillermo; Crespo, Julio; Lagomarsino Montoya, Mario; Peña Testa, Claudia. (2018). Salud mental y la sociedad abierta en la realidad actual: entre la Utopía de la Cordura y la Praxis de la Locura. Revista Internacional de Filosofía y Teoría Social , 42- 49.

- https://medlineplus.gov/spanish/mentalhealth.html. (16 de Julio de 2018). Obtenido de Medlineplus: https://medlineplus.gov/spanish/mentalhealth.html

- Nami National Alliance con Mental Illness. (s.f.). Obtenido de https://www.nami.org/: https:// www.nami.org/Find-Support/Diverse-Communities/Latino-Mental-Health/La-salud-mentalen-la-comunidad-latin

- $\quad$ Oramas Viera, Arlene; Santana López, Sandra; Vergara Barrenechea, Adriana. (2006 ). El bienestar psicologico, un indicador positivo de la salud mental . Revista Cubana de Salud y Trabajo. , 34 a 39.

- Proceso de Amparo, Azanca con Ministerio de Salud (Triibunal Constitucional Peruano 2003).

- Puga, D. (Mayo - Junio de 2005). La dependencia de las personas con discapacidad: entre lo sanitario y lo social, entre lo privado y lo público. Revista Especializada en Salud Pública, 327 a 330.

- $\quad$ R.J.S.A. Vda de R con Seguro Social de Salud, Tribunal Constitucional (2007).

- Rondón, M. (2006). Salud mundial: un problema de salud pública en el Perú. Revista Peruana de Medicina Experimental y Salud Pública., 23, 237-238.

- Ticona Cano, P. E. (2014 ). El derecho a la salud mental en el Perú del siglo XXI ¿un derecho protegido o un derecho postergado por el Estado peruano? Tesis para optar el grado académico de Magister en Derecho Constitucional de la Pontificia Católica del Perú .

- Valencia Collazos, M. (2007). Trastornos mentales y problemas de salud mental. Día mundial de la salud mental. Salud Mental, 30, 75 - 80. Obtenido de Instituto Nacional de Psiquiatría Ramón de la Fuente Muñiz: http://repositorio.inprf.gob.mx/handle/123456789/7151

- Vélez Arango , A. (2007). Nuevas dimensiones del concepto de salud: El derecho a la saluid en el Estado social de Derecho. Revista Hacia la Promoción de la Salud. 